\title{
A Flexible Domain-domain Hinge Promotes an Induced-fit Dominant Mechanism for the Loading of Guide-DNA into Argonaute Protein in Thermus thermophilus
}

Lizhe $\mathrm{Zhu}^{1,2}$, Hanlun Jiang ${ }^{2,3}$, Fu Kit Sheong ${ }^{1}$, Xuefeng Cui ${ }^{4}$, Xin $\mathrm{Gao}^{4}$, Yanli Wang ${ }^{5}$ and Xuhui Huang ${ }^{1,2,3 *}$

${ }^{1}$ Department of Chemistry, ${ }^{2}$ Center of Systems Biology and Human Health, School of

Science and Institute for Advance Study, ${ }^{3}$ Bioengineering Graduate Program, The

Hong Kong University of Science and Technology, Clear Water Bay, Kowloon, Hong

Kong

${ }^{4}$ Computer, Electrical and Mathematical Sciences and Engineering Division, King

Abdullah University of Science and Technology, Thuwal 23955-6900, Saudi Arabia

${ }^{5}$ Laboratory of Non-Coding RNA, Institute of Biophysics, Chinese Academy of

Sciences, Beijing 100101, China;

*To whom correspondence should be addressed. xuhuihuang@ust.hk 


\section{Contents}

SI Fig.1 Direct contacts between PAZ and PIWI domain in the binary crystal of TtAgo.

SI Fig.2 Unbiased Molecular Dynamics simulation of apo TtAgo started from four crystal structures.

SI Fig.3 Definition of $d_{\text {PAZ-PIWI }}$ - distance between PAZ and PIWI tip.

SI Fig.4 Comparison of the PIWI domain between hAgo2 and TtAgo.

SI Fig.5 Free energy surface of $d_{\mathrm{PAZ}-\mathrm{PIWI}}$ and number of salt-bridges between PAZ and other domains for TtAgo and hAgo2.

SI Tab.1 Unambiguous distance restraints for HADDOCK simulations. 


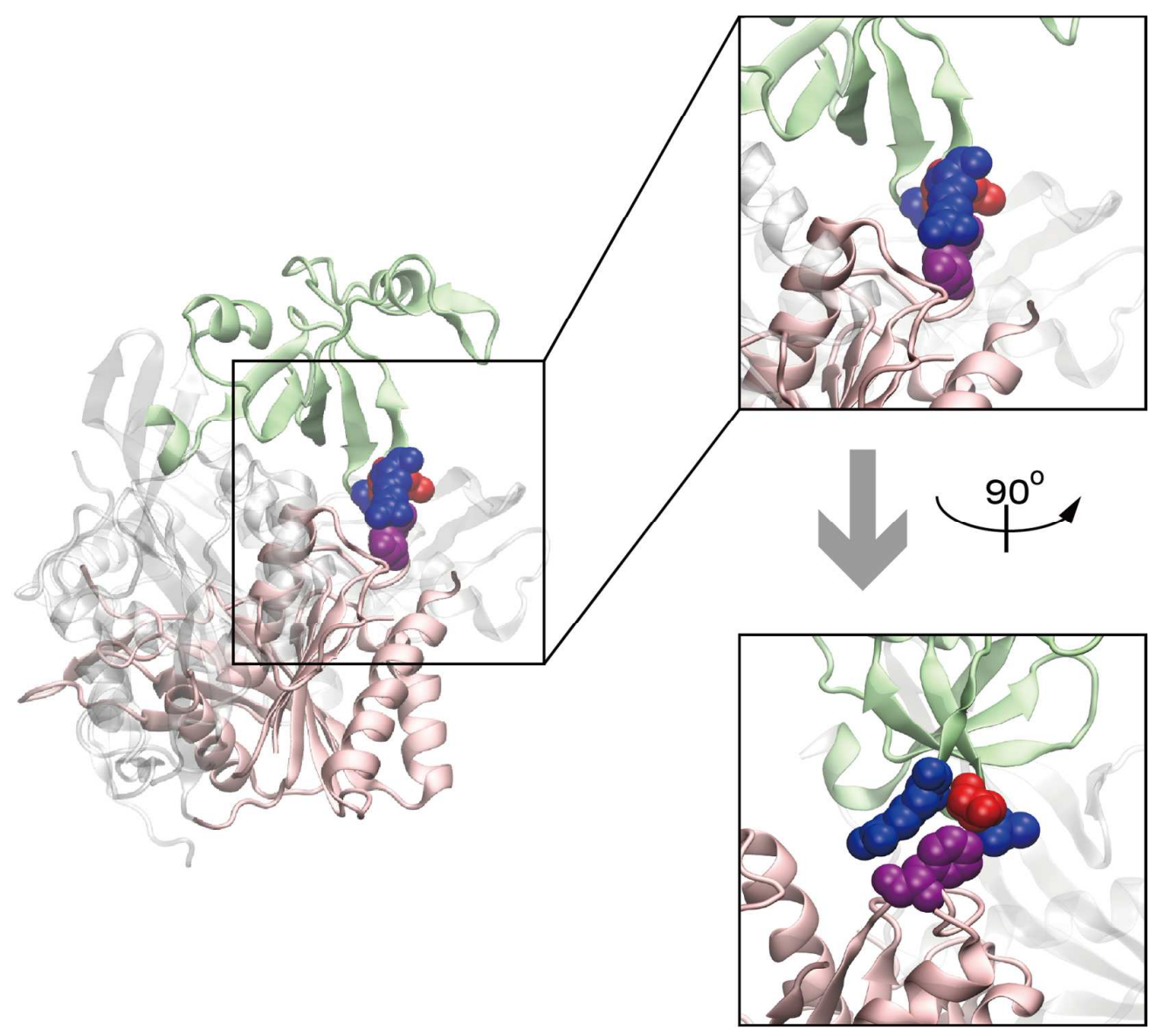

Figure 1. Direct contacts between PAZ and PIWI domain in the binary crystal structure of TtAgo. PAZ and PIWI are colored green and pink respectively. Residues that contribute to the contacts are visualized in VMD representation: blue/red - PAZ residues; purple - PIWI residues. 

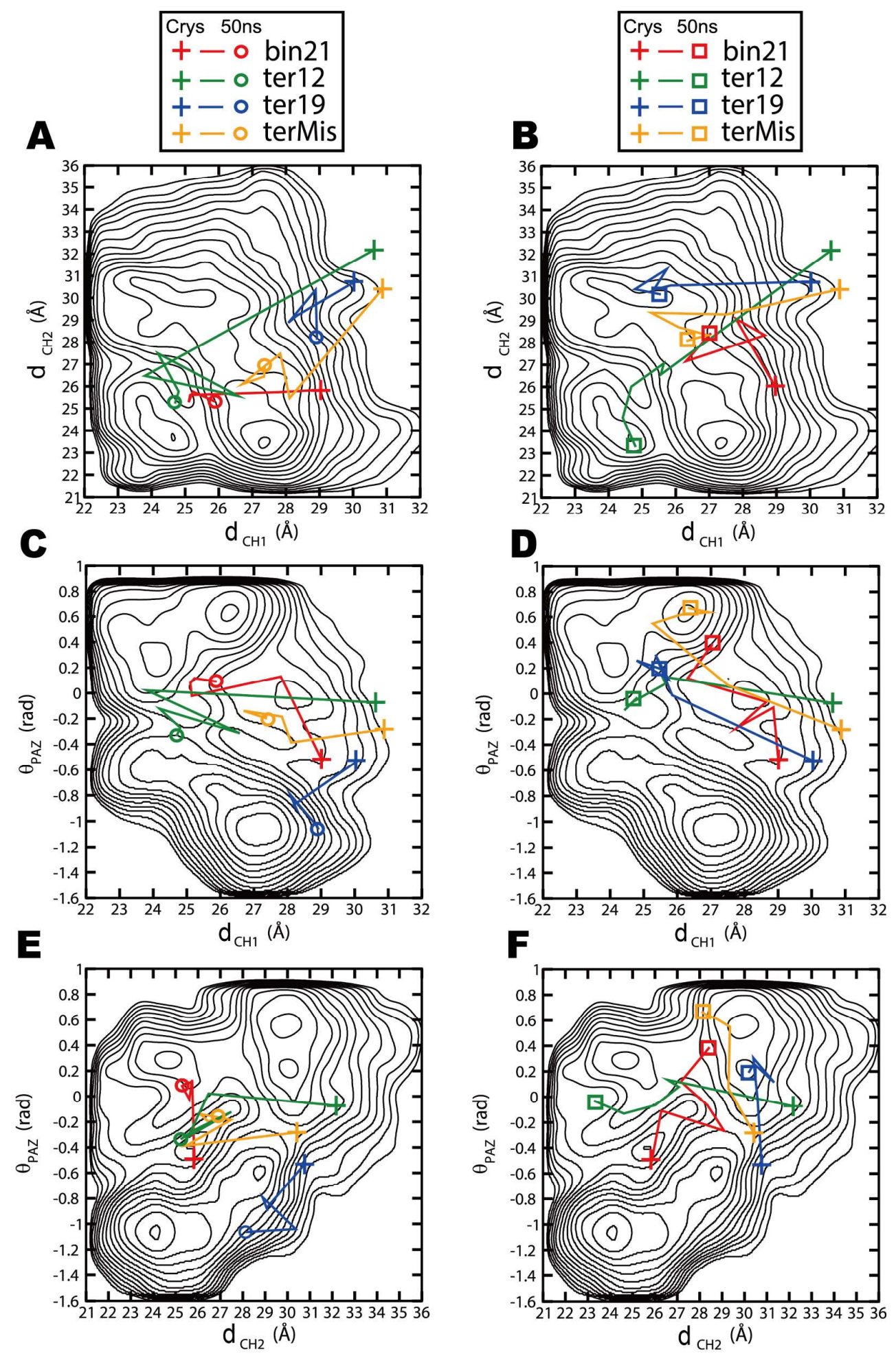

Figure 2. Unbiased Molecular Dynamics simulation of apo TtAgo starting from four crystal structures. All trajectories were 50ns long and projected on CV space. (A)(B) $\mathrm{d}_{\mathrm{CH} 1}$ and $\mathrm{d}_{\mathrm{CH} 2}(\mathbf{C})(\mathbf{D}) \mathrm{d}_{\mathrm{CH} 1}$ and $\theta_{\mathrm{PAZ}}(\mathbf{E})(\mathbf{F}) \mathrm{d}_{\mathrm{CH} 2}$ and $\theta_{\mathrm{PAZ}}$. Two trajectories were performed for each crystal. $(\mathbf{A})(\mathbf{C})(\mathbf{E})$ correspond to Trajectory 1, (B)(D)(F) correspond to Trajectory 2 . 


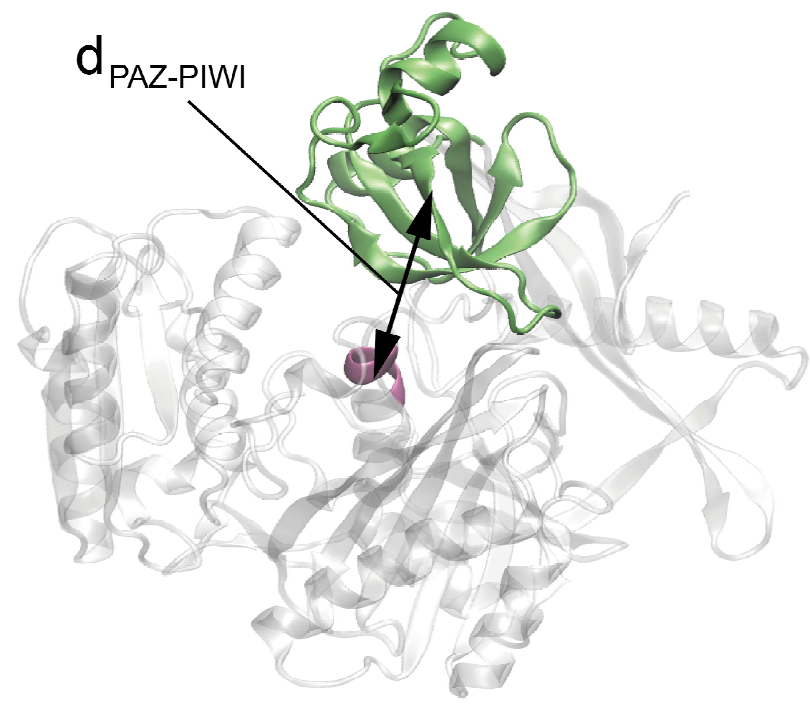

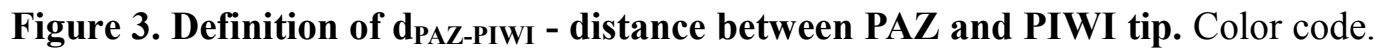
green - PAZ, magenta - PIWI tip.

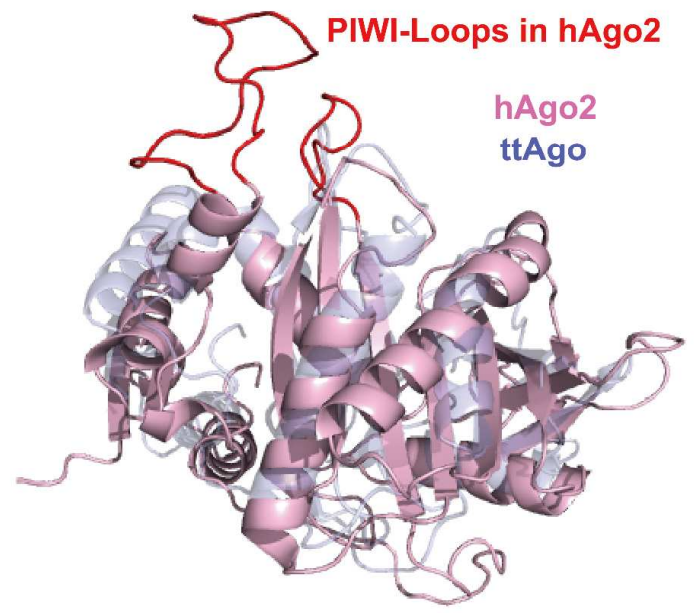

Figure 4. Comparison of the PIWI domain between hAgo2 and TtAgo. hAgo2 contains two long loops that do not exist in TtAgo. Role of such long loops in regulating miRNA loading into hAgo 2 has been discussed in our previous work ${ }^{1}$. 

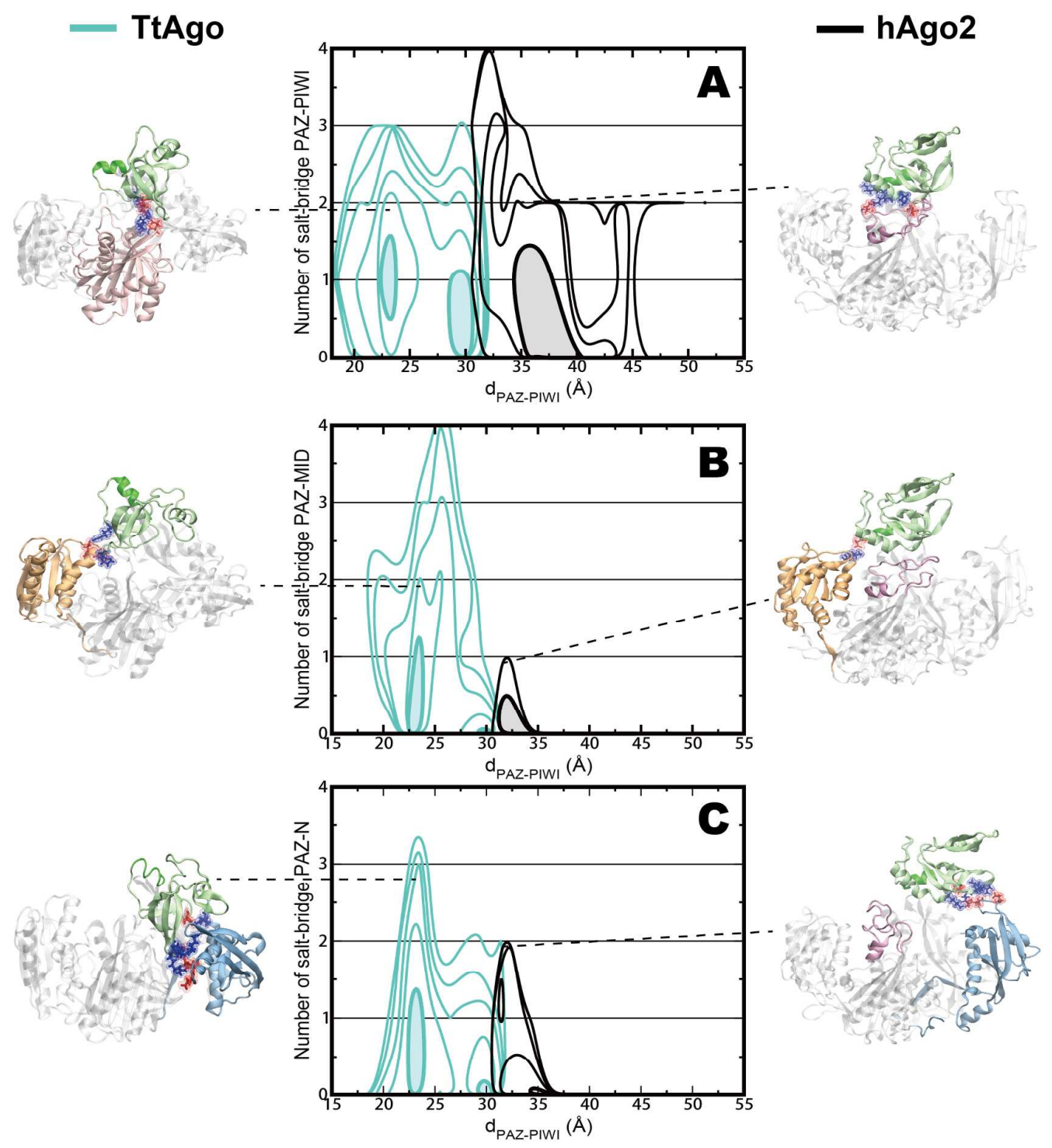

Figure 5. Free energy surface of $d_{\mathrm{PAZ}-\mathrm{PIWI}}$ and number of salt-bridges between PAZ and other domains for TtAgo and hAgo2. $\mathrm{d}_{\text {PAZ-PIWI }}$ (see S4 Fig) is center of mass distance between PAZ and segment of PIWI domain. Contour lines are plotted every $1 \mathrm{k}_{\mathrm{B}} \mathrm{T}$ and up to $5 \mathrm{k}_{\mathrm{B}} \mathrm{T}$. Representative structures of both Agos are visualized. In general, PAZ is closer to PIWI, N and MID in TtAgo than in hAgo2. From (A), it appears that more salt-bridges are formed between PAZ and PIWI in hAgo2 than in TtAgo. However, note that the PIWI residues contributing to the salt-bridges in hAgo2, these residues are all located at the two long loops on PIWI, which is absent in TtAgo. In fact, more salt-bridges are found in TtAgo than in hAgo2 (B) between PAZ and MID (C) between PAZ and N. In TtAgo, these salt-bridges stabilize the contacts between PAZ and other domains and therefore facilitate blocking of the nucleic-acid-binding-channel. Salt-bridge analysis is performed via $\mathrm{VMD}^{2}$ with cut-off distance of 3.2 Å between the Oxygen and Nitrogen atoms. 


\begin{tabular}{|l|l|l|l|l|l|}
\hline \multicolumn{2}{|c|}{ Ago } & \multicolumn{2}{c|}{ DNA } & $\begin{array}{c}\text { Distance } \\
(\AA)\end{array}$ & $\begin{array}{c}\text { Error } \\
\text { range }(\AA)\end{array}$ \\
\hline $\begin{array}{l}\text { Residue } \\
\text { ID }\end{array}$ & Atom name & Residue ID & Atom name & \multicolumn{1}{|c|}{} \\
\hline K422 & NZ & T1 & P & 3.0 & \pm 0.5 \\
\hline R446 & CZ & G2 & P & 4.0 & \pm 0.5 \\
\hline K230 & NZ & G17 & P & 4.0 & \pm 0.5 \\
\hline R232 & CZ & T18 & P & 3.5 & \pm 0.5 \\
\hline H227 & NE2 & T18 & H3T & 1.5 & \pm 0.5 \\
\hline
\end{tabular}

Table 1. Unambiguous distance restraints for HADDOCK simulations. The restraints were selected from the closest contacts between Ago and terminal nucleotides of DNA in the crystal structure (PDB ID. 3DLH). 


\section{References}

(1) Jiang, H.; Sheong, F. K.; Zhu, L.; Gao, X.; Bernauer, J.; Huang, X. Markov State Models Reveal a Two-step Mechanism of miRNA Loading into the Human Argonaute Protein: Selective Binding Followed by Structural Re-arrangement. PLoS Comput. Biol. 2015, 10.1371/journal.pcbi.1004404.

(2) Humphrey, W.; Dalke, A.; Schulten, K. VMD: Visual Molecular Dynamics. J. Mol. Graph. 1996, 14, 33-38, 27-38. 